\title{
SOFT SKILLS NA ENGENHARIA: UM RELATO DE CASO DO ICT/UNIFESP
}

Laura Fagnani-fagnani@unifesp.br

Instituto de Ciência e Tecnologia - UNIFESP - Campus São José dos Campos

Rua Talim, 330 - Vila Nair

12231-280 - São José dos Campos - São Paulo

Arthur Freitas Araújo - af.araujo@unifesp.br

Instituto de Ciência e Tecnologia - UNIFESP - Campus São José dos Campos

Rua Talim, 330 - Vila Nair

12231-280 - São José dos Campos - São Paulo

Verônica Ribeiro dos Santos - veronica.ribeiro@unifesp.br

Instituto de Ciência e Tecnologia - UNIFESP - Campus São José dos Campos

Rua Talim, 330 - Vila Nair

12231-280 - São José dos Campos - São Paulo

Mariana Motisuke-motisuke@unifesp.br

Instituto de Ciência e Tecnologia - UNIFESP - Campus São José dos Campos

Rua Talim, 330 - Vila Nair

12231-280 - São José dos Campos - São Paulo

Resumo: O termo VUCA foi criado nos anos 1990 para descrever como o mundo ficou depois do final da guerra fria: Volátil, incerto (uncertainty), complexo e ambíguo. Esse termo não poderia ser mais recente. Afinal, as coisas mudam muito rápido, sem que saibamos para onde vão, sem que necessariamente entendamos o que causou esta mudança e o resultado nem sempre é simples de avaliar e processar. Este cenário gera instabilidade emocional nas pessoas, aumenta o nível de exigência do mercado de trabalho e, como consequência, vivenciamos elevados níveis de estresse e de insatisfação com a vida pessoal e profissional. Para lidar de maneira saudável e equilibrada com as novas exigências de um mundo cada vez. mais VUCA, o desenvolvimento de competências socioemocionais e comportamentais (soft skills) torna-se relevante, principalmente para os jovens adultos, entre 15 e 30 anos, que irão vivenciar os maiores impactos do mundo moderno. Como forma de fomentar o desenvolvimento de competências que auxiliem os estudantes a navegar em um mundo cada vez mais instável e complexo, algumas universidades estão incluindo em suas grades curriculares disciplinas que foquem no desenvolvimento destas competências. Este trabalho tem como objetivo relatar o caso da implementação do ensino de soft skills no curso de engenharia de materiais do ICT/UNIFESP.

Palavras-chave: Soft Skills. Inovação no ensino. Curricularização da extensão. 


\section{INTRODUÇÃO}

A denominada Quarta Revolução Industrial (SCHWAB, 2016) vem trazendo imensuráveis avanços para a sociedade, para a economia e para a saúde, porém se diferenciando das três revoluções precedentes, pela velocidade do seu desenvolvimento tecnológico, evoluindo de maneira exponencial ao invés de linear. É uma transformação que se constrói pela junção de tecnologias e pelo estreitamento entre as esferas física, biológica e digital (XU; DAVID; KIM, 2018). A rapidez com que estas mudanças acontecem geram incertezas, complexidade e ambiguidade, tornando o mundo, portanto, cada vez mais VUCA (BENNETT; LEMOINE, 2014).

Este termo, VUCA, criado nos anos 1990 para descrever como o mundo ficou depois do final da guerra fria e não poderia ser mais recente. Tudo muda muito rápido (volatile), sem que saibamos para onde vão (uncertainty), sem que necessariamente entendamos o que causou esta mudança (complexity) e o resultado nem sempre é simples de avaliar e processar (ambiguity). Este cenário gera instabilidade emocional nas pessoas, aumenta o nível de exigência do mercado de trabalho (problemas cada vez mais dinâmicos, complexos e interdisciplinares) e, como consequência, vivenciamos elevados níveis de estresse e de insatisfação com a vida pessoal e profissional (BENNETT; LEMOINE, 2014; SEOW; PAN; KOH, 2019).

As mudanças com relação à indústria e ao cenário mundial impactaram diretamente no mundo do trabalho, acarretando o desaparecimento de profissões já obsoletas, o surgimento de ocupações mais atualizadas e mudanças nas competências demandadas ao profissional contemporâneo (BERLINGERI, 2018) e, também, repercutindo no panorama do engenheiro atual. Durante mais de dois séculos, os papéis que um engenheiro poderia desempenhar e o quê a formação em Engenharia deveria ensinar estavam relacionados fortemente ao país de atuação desse profissional e a conhecimentos técnicos específicos a cada área (FILHO et al., 2019; LUCENA et al., 2008). Hoje, o mercado demanda uma maior articulação dos conhecimentos e o uso competências que não são desenvolvidas no dia a dia universitário. Este cenário criou uma lacuna entre o perfil do egresso dos cursos de engenharia e o perfil profissional demandado pelo mercado (BERLINGERI, 2018).

O mundo VUCA não impactou somente o mercado de trabalho, significativas mudanças também atingiram a sociedade e a saúde. O excesso de informação, a grande velocidade de mudança e as incertezas do mundo moderno tem resultado em um aumento no número de casos de doenças psicológicas como depressão, burnout, ansiedade e pânico. De fato, se olharmos para os dados publicados pela Our World in Data, em 2017 eram 3,87\% da população mundial sofrendo com transtornos de ansiedade. No Brasil este número é ainda mais preocupante, $6,45 \%$, ou seja, mais de 13 milhões de pessoas sofrem com transtornos de ansiedade. Ainda, observando a prevalência dos transtornos de ansiedade por faixa etária temos: 6,33\% dos jovens 15-19 anos; 7,08\% entre 20-24 anos; 7,63 entre 25-29 anos e; 8,03\% entre 30-34 anos (DATA, $\left[\begin{array}{ll}s . d .\end{array}\right]$, são números bastante preocupantes.

Felizmente, a literatura tem mostrado que o desenvolvimento de soft skills desempenha um importante papel em questões sociais, econômicas e de saúde (CAZAN; NĂSTASĂ, 2015; DIREITO; PEREIRA; DUARTE, 2012; JAMESON et al., 2016; KASHANI; AZIMI; VAZIRI, 2012; KUMAR; HSIAO, 2007; PULKO; PARIKH, 2003; SEOW; PAN; KOH, 2019). Existem várias evidências da relação entre as soft skills e o rendimento escolar (JENAABADI, 2014; KASHANI; AZIMI; VAZIRI, 2012), a saúde mental (MARTINS; RAMALHO; MORIN, 2010) e o bem-estar e a satisfação com a vida (CAZAN; NĂSTASĂ, 2015; MIKOLAJCZAK; VAN BELLEGEM, 2017). Soma-se a isso a clara a evidência da importância dessas competências na inserção no mercado de trabalho, previsão salarial e outras questões 
relacionadas ao sucesso profissional, como no gerenciamento de mudanças e do estresse no ambiente de trabalho, satisfação com a carreira e maior rendimento (HENDARMAN; TJAKRAATMADJA, 2012; SCHUTTE; LOI, 2014; SEOW; PAN; KOH, 2019; UDAYAR et al., 2018), principalmente em um contexto mundial de mudanças rápidas, incertezas e elevada complexidade.

Como forma de fomentar o desenvolvimento de competências que auxiliem os estudantes a navegarem em um mundo cada vez mais instável e complexo, algumas universidades estão incluindo em suas grades curriculares disciplinas que focam no desenvolvimento destas competências baseando-se, por exemplo, nos conceitos da psicologia positiva, no desenvolvimento das competências da inteligência emocional e em metodologias ativas de ensino-aprendizagem. A disciplina Psychology of the Good Life criada em 2018 em Yale ensina ferramentas para o desenvolvimento de bem-estar emocional baseado nas teorias da psicologia positiva, foram mais de 1000 estudantes inscritos em sua primeira turma. Outros exemplos são a disciplina Design Thinking to the wicked problems of creating fulfilling lives and careers que foi criada com o propósito de ajudar os estudantes de Stanford com questões emocionais relacionadas às vidas pessoais e profissionais. E, também em Stanford, a disciplina Designing your life foi criada por Bill Burnet e Dave Evans (SHIMER, 2018).

O Brasil não fica para trás, também em 2018 a UnB ofereceu pela primeira vez uma disciplina focada em melhoria de bem-estar para estudantes de engenharia (Tópicos especiais em engenharia: felicidade) em que a ementa traz os conceitos da psicologia positiva e algumas ferramentas de coaching e mentoria para auxiliarem os estudantes a lidarem com o dia a dia universitário (GARONCE, 2018). Em 2019, no Instituto de Ciência e Tecnologia da UNIFESP (ICT/UNIFESP) a unidade curricular Tópicos Especiais em Engenharia de Materiais I: Soft Skills na Engenharia tem como principal enfoque mostrar a importância que competências socioemocionais e comportamentais têm na vida pessoal e profissional e como elas podem ser desenvolvidas de forma autônoma.

A inteligência emocional (IE) é a base das competências socioemocionais e muitos estudos mostram que elevados níveis de IE estão associados com baixos níveis de ansiedade, estresse e burnout e maiores níveis de satisfação com a vida, sucesso acadêmico e profissional (CAZAN; NĂSTASĂ, 2015; DI FABIO; SAKLOFSKE, 2014; JAMESON et al., 2016; KASHANI; AZIMI; VAZIRI, 2012). A capacidade de reconhecer, entender e gerenciar as emoções nos níveis individual e social, em quaisquer situações é a premissa base da IE. O quociente emocional de um indivíduo é composto por um conjunto de habilidades que definem como a pessoa se sente em relação a ela mesma, como ela lida com o que acontece em seu ambiente exterior e como ela se relaciona com os outros (TIBA; MOTISUKE, 2016). Tendo todas essas habilidades desenvolvidas e em equilíbrio a pessoa lida melhor com as instabilidades geradas pelo seu ambiente externo e sente-se mais realizada tanto pessoalmente quanto profissionalmente (SCHUTTE; LOI, 2014).

Em conjunto com as competências socioemocionais estão as comportamentais que englobam disciplinas como liderança, trabalho em equipe, responsabilidade social, ética, gestão do tempo, comunicação eficaz, empatia, otimismo e pensamento criativo. São competências que se desenvolvem a partir das experiências de vida do indivíduo, mas que podem ser desenvolvidas em jovens quando eles são expostos aos estímulos corretos como por exemplo em disciplinas que utilizem metodologias ativas de ensino-aprendizagem.

Desta forma, este trabalho tem como objetivo relatar a experiência de implementação do ensino de soft skills na Engenharia de Materiais (EM) do Instituto de Ciência e Tecnologia da Universidade Federal de São Paulo, campus São José dos Campos - ICT/UNIFESP. 


\section{DESCRIÇÃO DA UNIDADE CURRICULAR E DA METODOLOGIA DE ENSINO- APRENDIZAGEM ADOTADA EM SALA DE AULA}

Soft Skills na Engenharia (SSEN) é uma unidade curricular (UC) eletiva da Engenharia de Materiais do ICT/UNIFESP que foi criada no $1^{\circ}$ semestre de 2019. O contexto para a sua criação foi levantado na introdução deste trabalho e, desta forma, os objetivos da UC são:

1) Conscientizar o estudante da importância das soft skills para a sua inserção no mercado possibilitando o trabalho colaborativo, a liderança e a integração de equipes multidisciplinares, a comunicação efetiva com o cliente, a negociação, a tomada de decisão e, o gerenciamento do estresse;

2) Conscientizar da importância destas competências para o gerenciamento de questões pessoais e para uma melhora na qualidade de vida e do bem-estar e;

3) Fomentar o autodesenvolvimento pessoal do estudante nas competências e técnicas apresentadas.

A UC foi elaborada com uma carga horária de 72 horas (4 horas semanais) sendo 36 horas teóricas e 36 horas de extensão, articuladas com o projeto de extensão universitária "Desenvolvendo Soft Skills". Esta ação tem como principal objetivo divulgar para jovens (entre 15 e 30 anos), que estão iniciando a vida acadêmica e/ou profissional, a importância e o conhecimento sobre estas competências que estão ganhando cada vez mais importância. A cada semestre, os estudantes ficam imersos em temas relacionados às soft skills e elaboram materiais educativos que possam ser utilizados em oficinas gratuitas e abertas à comunidade ou em divulgações digitais.

A UC tem sido oferecida todos os semestres desde a sua primeira oferta e o perfil de estudantes matriculados mudou bastante. Atualmente, estão matriculados estudantes de quase todos os cursos de graduação do ICT/UNIFESP e o contexto de aplicação das competências e as atividades desenvolvidas foram adaptadas a um contexto mais geral.

As competências abordadas na UC são escolhidas a cada semestre de acordo com o perfil dos estudantes matriculados, pesquisas na literatura e feedbacks recebidos dos semestres anteriores. A Tabela 1 descreve o conjunto de competências discutido a cada semestre e resume observações que guiaram as mudanças para os semestres seguintes.

Tabela 1 - Conjunto de competências abordadas a cada semestre e observações que guiaram as escolhas a cada semestre.

\begin{tabular}{|c|c|c|c|}
\hline Semestre & $\begin{array}{l}\text { Perfil dos } \\
\text { estudantes } \\
\text { matriculados }\end{array}$ & Conteúdo & Observações e mudanças \\
\hline $\begin{array}{c}1^{\mathbf{o}} \\
\text { semestre } \\
2019\end{array}$ & $\begin{array}{l}\text { Majoritariamente } \\
\text { estudantes } \\
\text { regulares da } \\
\text { engenharia de } \\
\text { materiais ou que } \\
\text { estavam seguindo } \\
\text { a trajetória da } \\
\text { engenharia de } \\
\text { materiais do } \\
\text { bacharelado em }\end{array}$ & $\begin{array}{l}\text { 1 - Liderança; } \\
2 \text { - Inteligência } \\
\text { Emocional; } \\
3 \text { - Resiliência; } \\
4 \text { - Agilidade Emocional; } \\
5 \text { - Foco; } \\
6 \text { - Empatia; } \\
7 \text { - Relacionamentos; } \\
8 \text { - Otimismo e bem-estar } \\
\text { como competência; }\end{array}$ & $\begin{array}{l}1 \text { - muitos temas semelhantes } \\
\text { e poucas oportunidades de } \\
\text { desenvolvimento/aplicação } \\
\text { dos conhecimentos. } \\
2 \text { - Nos próximos semestres } \\
\text { focar em um número menor } \\
\text { de competências (ou grupo } \\
\text { de competências) e } \\
\text { proporcionar atividades que } \\
\text { levem à reflexão, }\end{array}$ \\
\hline
\end{tabular}




\begin{tabular}{|c|c|c|c|}
\hline & $\begin{array}{l}\text { ciência e } \\
\text { tecnologia }\end{array}$ & $\begin{array}{l}9 \text { - Comunicação efetiva; } \\
10 \text { - Empreendedorismo. }\end{array}$ & $\begin{array}{l}\text { autoconhecimento e } \\
\text { desenvolvimento ativo das } \\
\text { competências }\end{array}$ \\
\hline $\begin{array}{c}2^{\circ} \\
\text { semestre } \\
\text { de } 2019\end{array}$ & & $\begin{array}{l}1 \text { - Inteligência } \\
\text { Emocional/ Equilíbrio } \\
\text { Emocional/ Agilidade } \\
\text { Emocional } \\
2 \text { - Liderança } \\
3 \text { - Comunicação efetiva } \\
4 \text { - } \\
\text { Relacionamentos/empatia; } \\
5 \text { - Empreendedorismo }\end{array}$ & $\begin{array}{l}1 \text { - Faltou o olhar para a } \\
\text { sociedade, o atuar de forma } \\
\text { responsável e ética } \\
2 \text { - Trazer/discutir mais o } \\
\text { contexto real de aplicação } \\
\text { das competências }\end{array}$ \\
\hline $\begin{array}{c}1^{\circ} \\
\text { semestre } \\
\text { de } 2020 \\
\text { (atual) }\end{array}$ & & $\begin{array}{l}1 \text { - Inteligência Emocional } \\
2 \text { - Liderança } \\
3 \text { - Comunicação efetiva } \\
4 \text { - } \\
\text { Relacionamentos/empatia } \\
5 \text { - Empreendedorismo } \\
6 \text { - Responsabilidade } \\
\text { social }\end{array}$ & $\begin{array}{l}1 \text { - Muitos estudantes } \\
\text { matriculados com perfil mais } \\
\text { diversificado que o semestre } \\
\text { anterior. } \\
\text { 2- Semestre suspenso por } \\
\text { conta da pandemia de } \\
\text { COVID-19, com substituição } \\
\text { por atividades remotas. }\end{array}$ \\
\hline
\end{tabular}

Fonte: elaboração própria

Além das competências abordadas de forma explícita, outras competências foram desenvolvidas ao longo das atividades a saber: pensamento crítico, pensamento criativo, trabalho colaborativo e autoconhecimento.

\subsection{Metodologia de ensino-aprendizagem adotada}

A UC foi organizada de forma a proporcionar o conhecimento teórico sobre os temas abordados (competências) e vivências ou dinâmicas capazes de trazer a reflexão e a percepção sobre a proficiência em cada competência. Além disso, ao final de cada tema, discutia-se maneiras de se desenvolver determinada competência.

As metodologias de ensino-aprendizagem empregadas ao longo de todo o semestre foram majoritariamente ativas/imersivas com alguns momentos expositivos, mas os estudantes foram os protagonistas do próprio aprendizado, mesmo durante a retomada do $1^{\circ}$ semestre de 2020 de forma retoma. Foram utilizadas ferramentas digitais para facilitação online. As principais metodologias utilizadas foram:

\section{Discussões em grupos}

Em várias aulas os estudantes são divididos em grupos para discutir sobre um tema ou pergunta, criar uma opinião comum e depois apresentar essa opinião para seus pares e ao final ampliar o diálogo e a compreensão sobre o tema.

Por exemplo, neste $1^{\circ}$ semestre de 2020, a turma tem um perfil bastante diverso com estudantes da engenharia de materiais, engenharia de computação, biotecnologia, ciência e tecnologia e engenharia biomédica. E os impactos da indústria 4.0 e da revolução digital na carreira de cada um deles são bem distintos. Assim, eles se agruparam por afinidade de atuação profissional e discutiram sobre o futuro do emprego e a indústria 4.0 sob a perspectiva de sua área. Em seguida fizeram um breve brainstorming, utilizando uma folha de flipchart e post-its, 
sobre as competências ou conhecimentos mais importantes para se manter bem colocado no mercado em um cenário de 5 a 10 anos. Finalmente, os estudantes deveriam agrupar esses conhecimentos e competências e preparar uma breve fala sobre a conclusão do grupo. Ao final de cada apresentação, uma discussão era conduzida e para fechar o tema, foi feita um mural com as competências e conhecimentos comuns e as específicas para cada área. A figura 1 ilustra essas atividades de discussão em grupos.

Figura 1 - Discussões em grupo em uma das turmas de Soft Skills na Engenharia.

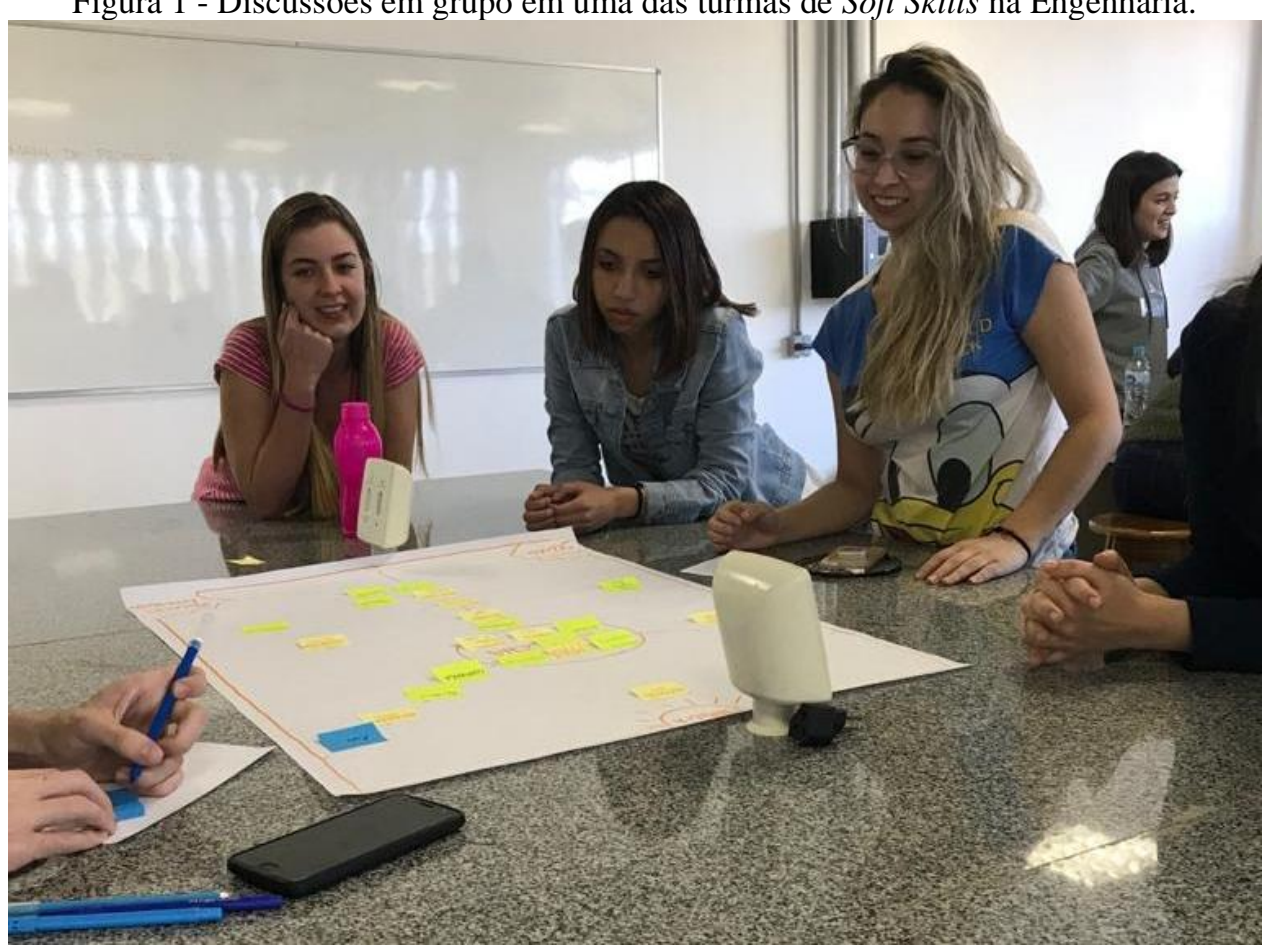

Fonte: o próprio autor

\section{Encenações (role-plays)}

Em uma das aulas sobre inteligência emocional (IE), os estudantes receberam um handout contento as 12 dimensões da IE, a saber: consciência emocional, autoestima, senso de propósito, independência emocional, controle de impulsos, teste de realidade, flexibilidade, resiliência, expressão emocional, empatia, relacionamentos interpessoais e consciência social. Após ler o material individualmente, os alunos foram divididos em grupos e escolheram uma dimensão. Em seguida, tiveram que criar uma encenação que de alguma forma representasse a dimensão escolhida. Após cada apresentação foi feita uma discussão para entender qual era a dimensão que a situação encenada representava e qual tinha sido a percepção de quem assistiu sobre a situação.

\section{Dinâmicas em grupo}

Em alguns momentos os alunos participam de dinâmicas de grupo em que eles vivenciam algumas situações e refletem sobre as competências que faltaram ou que precisaram utilizar. Toda dinâmica é finalizada com uma discussão para validar a percepção de todos e refletir sobre formas de agir e/ou desenvolver uma determinada competência. 
Uma dinâmica bastante utilizada é a da construção de uma torre de macarrão em que os alunos em grupos têm alguns espaguetes, fita adesiva e barbante, em quantidades limitadas e devem construir uma torre alta e estável em 25 minutos. A equipe vencedora é aquela que construir a torre mais alta e que sustente, sem quebrar, o peso de um elemento de teste. Ao longo da dinâmica, algumas situações são colocadas como mudança de equipe, redução de orçamento e/ou do tempo de entrega. A figura 2 ilustra a dinâmica em andamento em uma das turmas da UC. Ao final da dinâmica, é feita uma roda de conversa para discutir como cada estudante se sentiu e como percebeu a situação.

$\mathrm{Na}$ aula sobre os estilos de liderança os estudantes simulam em grupos uma fábrica que tem um processo produtivo bem estabelecido. A cada rodada do processo um membro do grupo é o líder da equipe e deve liderar de acordo com um determinado estilo (coercivo, visionário, marcador de ritmo, democrático, etc.). Ao final é realizada uma discussão com toda a sala em que se reflete sobre quais as diferenças entre cada estilo de liderança e qual é mais ou menos efetivo e se há situações em que um determinado tipo de liderança é requerido.

Figura 2 - Dinâmica da torre de macarrão em uma das turmas de Soft Skills na Engenharia.

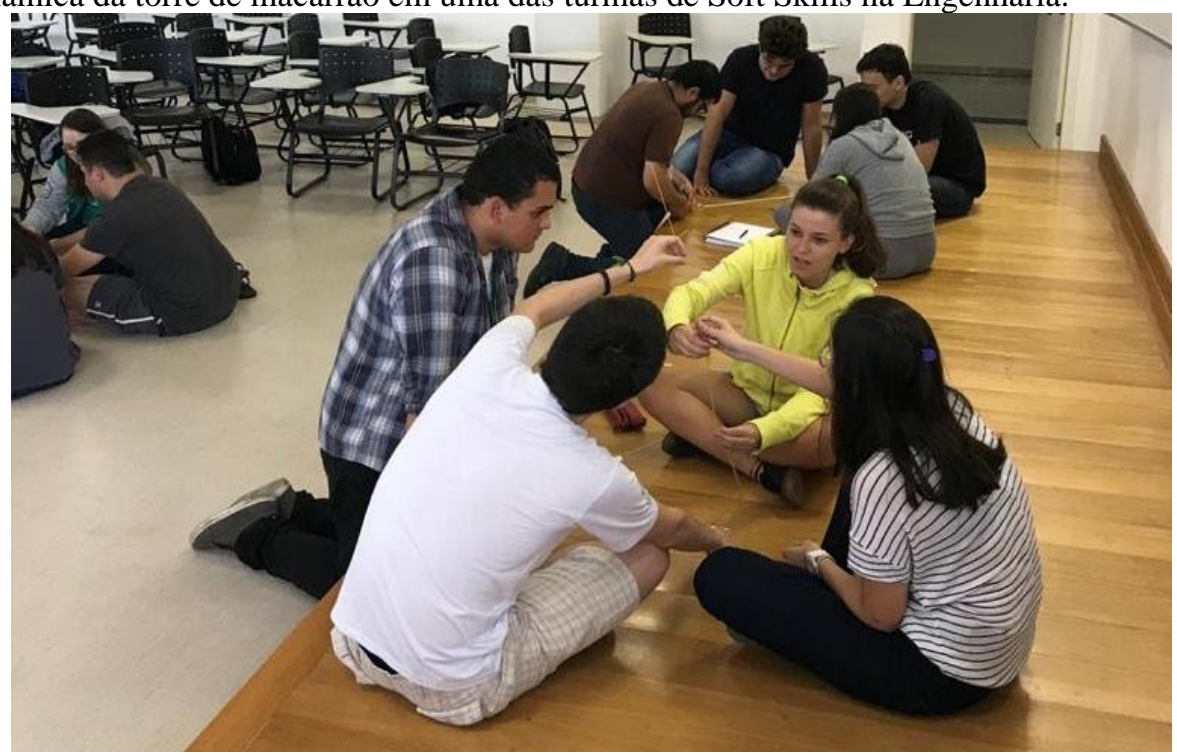

Fonte: o próprio autor

\section{Criação de conteúdos para a divulgação para a comunidade (extensão universitária)}

A cada semestre os estudantes precisam criar individualmente ou em grupo conteúdos sobre as competências discutidas ao longo do semestre. Este material é divulgado nas ações do projeto de extensão universitária Desenvolvendo Soft Skills. No $1^{\circ}$ semestre de 2019, os alunos criaram, individualmente, um breve discurso (5-10 minutos) sobre uma competência de escolha. Esta fala deveria ser parecida a um TED Talk e foi avaliada por uma banca de professores. Os três estudante que tiveram as falas mais bem avaliadas foram convidados para se apresentarem em uma palestra da II Semana de Promoção do Bem-estar do ICT/Unifesp.

Já no segundo semestre de 2019, os alunos criaram materiais/conteúdos em grupo para a divulgação das soft skills para a comunidade jovem. Esses materiais foram criados a partir de um processo de design thinking em que os alunos passaram por todas as etapas (empatia, definição, ideação, protótipo e validação) e criaram desde uma oficina sobre empatia até vídeos e podcast sobre autoestima. Os materiais audiovisuais foram divulgados nas redes sociais do projeto desenvolvendo soft skills. 
Esta articulação com o projeto de extensão é de extrema importância para fortalecer a relação universidade-comunidade e, também para criar no estudante a consciência de que ele pode impactar positivamente na comunidade em que vive.

\section{CONSIDERAÇÕES FINAIS}

O ensino de soft skills não pode mais ser deixado em segundo plano. O mundo está em constante transformação gerando instabilidades e necessidade de adaptação. $O$ desenvolvimento de competências soft pode ser favorecido durante a graduação de muitas formas e o exemplo da UC descrita neste trabalho é uma delas.

O interesse pela UC Soft Skills na Engenharia tem crescido a cada novo oferecimento e a diversidade de alunos matriculados também. Na primeira vez que foi oferecida a UC foram 20 matriculados e os estudantes em sua maioria eram da Engenharia de Materiais. Já no $2^{\circ}$ semestre de 2019 foram 36 matriculados e uma maior diversidade de cursos. Neste semestre, $1^{\circ}$ de 2020 , foram 32 matriculados tendo a participação de estudantes de quase todos os cursos de graduação do ICT/UNIFESP (eng. de materiais, eng. da computação, eng. biomédica, ciência da computação, biotecnologia e ciência e tecnologia).

Os feedbacks ao final de cada semestre têm sido positivos e os estudantes demonstram bastante engajamento ao longo das atividades e das aulas. Como exemplo, no $1^{\mathrm{o}}$ semestre de 2019, a UC teve 20 matriculados e os feedbacks foram bastante positivos, 14 alunos responderam a um questionário e $85 \%$ acharam os conteúdos importantes e relevantes para a vida pessoal e profissional; $57 \%$ se sente mais preparado para enfrentar os desafios do mercado de trabalho e $85 \%$ acharam que as atividades propostas, mesmo que às vezes desconfortáveis, foram importantes para o desenvolvimento pessoal e profissional.

Agora com a implementação das novas Diretrizes Curriculares Nacionais para os cursos de engenharia é primordial que os cursos encontrem formas para fomentar o desenvolvimento efetivo de soft skills em seus egressos para garantir uma efetiva inserção no mercado e uma maior satisfação com a vida.

\section{REFERÊNCIAS}

BENNETT, N.; LEMOINE, G. J. What a difference a word makes: Understanding threats to performance in a VUCA world. Business Horizons, vol. 57, no. 3, p. 311-317, 2014. DOI 10.1016/j.bushor.2014.01.001. Available at: http://dx.doi.org/10.1016/j.bushor.2014.01.001. BERLINGERI, M. M. Competências socioemocionais e mercado de trabalho: um estudo para o caso brasileiro. 2018. 59 f. Universidade de São Paulo, 2018. DOI 10.11606/D.96.2018.tde-17092018-115134. Available at: http://www.teses.usp.br/teses/disponiveis/96/96131/tde-17092018-115134/.

CAZAN, A.-M.; NĂSTASĂ, L. E. Emotional Intelligence, Satisfaction with Life and Burnout among University Students. Procedia - Social and Behavioral Sciences, vol. 180, no. November 2014, p. 1574-1578, 2015. DOI 10.1016/j.sbspro.2015.02.309. Available at: http://linkinghub.elsevier.com/retrieve/pii/S1877042815016559.

DATA, O. W. in. Prevalence of mental health disorders by disorder type. [s. d.]. Available at: https://ourworldindata.org/mental-health\#prevalence-of-mental-health-and-substance-usedisorders.

DI FABIO, A.; SAKLOFSKE, D. H. Promoting individual resources: The challenge of trait 
emotional intelligence. Personality and Individual Differences, vol. 65, p. 19-23, Jul. 2014.

DOI

10.1016/j.paid.2014.01.026.

Available

at:

http://linkinghub.elsevier.com/retrieve/pii/S0191886914000464.

DIREITO, I.; PEREIRA, A.; DUARTE, A. M. de O. Engineering Undergraduates' Perceptions of Soft Skills: Relations with Self-Efficacy and Learning Styles. Procedia - Social and Behavioral Sciences, vol. 55, p. 843-851, Oct. 2012. DOI 10.1016/j.sbspro.2012.09.571. Available at: http://dx.doi.org/10.1016/j.sbspro.2012.09.571.

FILHO, G. E.; SAUER, L. Z.; ALMEIDA, N. N. de; VILLAS-BOAS, V. Uma Nova Sala de Aula é Possível - Aprendizagem Ativa na Educação em Engenharia. 1st ed. [S. l.]: LTC, 2019.

GARONCE, L. UnB é primeira universidade pública do Brasil a oferecer disciplina sobre "felicidade." G1 Distrito Federal, 2018. Available at: https://g1.globo.com/df/distritofederal/noticia/2018/07/24/unb-e-primeira-universidade-publica-do-brasil-a-oferecerdisciplina-sobre-felicidade.ghtml.

HENDARMAN, A. F.; TJAKRAATMADJA, J. H. Relationship among Soft Skills, Hard Skills, and Innovativeness of Knowledge Workers in the Knowledge Economy Era. Procedia - Social and Behavioral Sciences, vol. 52, p. 35-44, 2012. DOI 10.1016/j.sbspro.2012.09.439. Available at: http://dx.doi.org/10.1016/j.sbspro.2012.09.439.

JAMESON, A.; CARTHY, A.; MCGUINNESS, C.; MCSWEENEY, F. Emotional Intelligence and Graduates - Employers' Perspectives. Procedia - Social and Behavioral Sciences, vol. 228, no. June, p. 515-522, 2016. DOI 10.1016/j.sbspro.2016.07.079. Available at: http://linkinghub.elsevier.com/retrieve/pii/S1877042816310059.

JENAABADI, H. Studying the Relation Between Emotional Intelligence and Self Esteem with Academic Achievement. Procedia - Social and Behavioral Sciences, vol. 114, no. 2004, p. 203-206, 2014. DOI 10.1016/j.sbspro.2013.12.685. Available at: http://linkinghub.elsevier.com/retrieve/pii/S187704281305324X.

KASHANI, F. L.; AZIMI, A. L.; VAZIRI, S. Relationship between Emotional Intelligence and Educational Achievement. Procedia - Social and Behavioral Sciences, vol. 69, no. Iceepsy, p. 1270-1275, 2012. DOI 10.1016/j.sbspro.2012.12.061. Available at: http://linkinghub.elsevier.com/retrieve/pii/S1877042812055218.

KUMAR, S.; HSIAO, J. K. Engineers Learn "Soft Skills the Hard Way": Planting a Seed of Leadership in Engineering Classes. Leadership and Management in Engineering, vol. 7, no. 1, p. 18-23, 2007. https://doi.org/10.1061/(ASCE)1532-6748(2007)7.

LUCENA, J.; DOWNEY, G.; JESIEK, B.; ELBER, S. Competencies Beyond Countries: The Re-Organization of Engineering Education in the United States, Europe, and Latin America. Journal of Engineering Education, vol. 97, no. 4, p. 433-447, Oct. 2008. DOI 10.1002/j.2168-9830.2008.tb00991.x. Available at: http://doi.wiley.com/10.1002/j.21689830.2008.tb00991.x.

MARTINS, A.; RAMALHO, N.; MORIN, E. A comprehensive meta-analysis of the relationship between Emotional Intelligence and health. Personality and Individual Differences, vol. 49, no. 6, p. 554-564, 2010. DOI 10.1016/j.paid.2010.05.029. Available at: http://dx.doi.org/10.1016/j.paid.2010.05.029.

MIKOLAJCZAK, M.; VAN BELLEGEM, S. Increasing emotional intelligence to decrease healthcare expenditures: How profitable would it be? Personality and Individual Differences, vol. 116, p. 343-347, 2017. DOI 10.1016/j.paid.2017.05.014. Available at: http://dx.doi.org/10.1016/j.paid.2017.05.014.

PULKO, S. H.; PARIKH, S. Teaching 'Soft' Skills to Engineers. International Journal of Electrical Engineering \& Education, vol. 40, no. 4, p. 243-254, 24 Oct. 2003. DOI 
10.7227/IJEEE.40.4.2. Available at: http://journals.sagepub.com/doi/10.7227/IJEEE.40.4.2. SCHUTTE, N. S.; LOI, N. M. Connections between emotional intelligence and workplace flourishing. Personality and Individual Differences, vol. 66, p. 134-139, 2014. DOI 10.1016/j.paid.2014.03.031. Available at: http://dx.doi.org/10.1016/j.paid.2014.03.031.

SCHWAB, K. The Fourth Industrial Revolution. 2016. Foreign Affairs. Available at: https://www.foreignaffairs.com/articles/2015-12-12/fourth-industrial-revolution.

SEOW, P. S.; PAN, G.; KOH, G. Examining an experiential learning approach to prepare students for the volatile, uncertain, complex and ambiguous (VUCA) work environment. International Journal of Management Education, vol. 17, no. 1, p. 62-76, 2019. DOI 10.1016/j.ijme.2018.12.001. Available at: https://doi.org/10.1016/j.ijme.2018.12.001.

SHIMER, D. Yale's Most Popular Class Ever: Happiness. New York Times, 2018. .

TIBA, R. M.; MOTISUKE, M. Introdução à Inteligência Emocional - Material de Treinamento. São José dos Campos: [s. n.], 2016.

UDAYAR, S.; FIORI, M.; THALMAYER, A. G.; ROSSIER, J. Investigating the link between trait emotional intelligence, career indecision, and self-perceived employability: The role of career adaptability. Personality and Individual Differences, vol. 135, no. April, p. 7-12, 2018. DOI 10.1016/j.paid.2018.06.046. Available at: https://doi.org/10.1016/j.paid.2018.06.046.

XU, M.; DAVID, J. M.; KIM, S. H. The Fourth Industrial Revolution: Opportunities and Challenges. International Journal of Financial Research, vol. 9, no. 2, p. 90, 5 Feb. 2018. DOI 10.5430/ijfr.v9n2p90. Available at: http://www.sciedupress.com/journal/index.php/ijfr/article/view/13194.

\title{
SOFT SKILLS IN ENGINEERING: A CASE REPORT FROM ICT/UNIFESP
}

\begin{abstract}
The acronym VUCA was created in the 1990s to describe what the world became after the end of the cold war: volatile, uncertain, complex and ambiguous. This word could not be more recent, after all, nowadays, things are changing very fast, without certain to where they are going, without necessarily understanding what caused it and the result is not always simple to evaluate and process. This scenario generates in people emotional instability, increases the level of the labor market demand and, as a consequence, we experience high levels of stress and dissatisfaction with personal and professional life. To deal with the new demands of an increasingly VUCA world in a healthy and balanced way, the development of socioemotional and behavioral skills (soft skills) becomes relevant, especially for young adults, between 15 and 30 years old, who will experience the greatest impacts of the modern world. As a way to foster the development of skills that help students navigate in an increasingly unstable and complex world, some universities are including disciplines that focus on the development of these skills in their curriculum. This work aims to report the case of the implementation of soft skills teaching in the materials engineering course at ICT / UNIFESP.
\end{abstract}

Keywords: soft skills. Innovation in education. Extension. 